Europhys. Lett., 68 (6), pp. 769-775 (2004)

DOI: $10.1209 /$ epl/i2004-10284-4

\title{
Propagation through fractal media: The Sierpinski gasket and the Koch curve
}

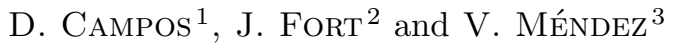 \\ 1 Grup de Física Estadística, Departament de Física \\ Universitat Autònoma de Barcelona - E-08193 Bellaterra, Barcelona, Spain \\ 2 Departament de Física, Universitat de Girona \\ Campus Montilivi, E-17071 Girona, Spain \\ 3 Departament de Medicina, Facultat de Ciències de la Salut \\ Universitat Internacional de Catalunya - c./Josep Trueta $s / n$ \\ E-08190 Sant Cugat del Vallès, Barcelona, Spain
}

received 7 April 2004; accepted in final form 18 October 2004

published online 30 November 2004

PACS. 05.40.-a - Fluctuation phenomena, random processes, noise, and Brownian motion.

PACS. 05.45.Df - Fractals.

\begin{abstract}
We present new analytical tools able to predict the averaged behavior of fronts spreading through self-similar spatial systems starting from reaction-diffusion equations. The averaged speed for these fronts is predicted and compared with the predictions from a more general equation (proposed in a previous work of ours) and simulations. We focus here on two fractals, the Sierpinski gasket (SG) and the Koch curve (KC), for two reasons, i.e. i) they are widely known structures and ii) they are deterministic fractals, so the analytical study of them turns out to be more intuitive. These structures, despite their simplicity, let us observe several characteristics of fractal fronts. Finally, we discuss the usefulness and limitations of our approach.
\end{abstract}

Introduction. - The analysis of transport processes on fractals received great attention during the early eighties [1], but in the last years it has decreased notably, although there are still many open problems in this field [2]. For instance, several attempts to describe diffusion in fractals by means of partial differential equations (PDEs) have been done [3-5]. Recently, we proposed [6] the Campos-Méndez-Fort (CMF) diffusion equation that accounts for the main scaling (power law) relations known. Moreover, that equation allows one to include into the formalism a growth function in order to consider reaction-diffusion (RD) systems, which are known to exhibit travelling front solutions [7]. By doing so, the characteristics of fronts spreading through fractal structures can also be studied, achieving again good agreement between this equation and previous theoretical results derived from scaling analysis [8].

The aim of this work is to show the interest and validity of some PDEs describing propagation within self-similar media, so they could be used in many potential applications as transport through porous soils [9], electronic properties of fractal structures [10], forest fires [11], biological invasions $[12], \ldots$. The main parameter which characterizes propagation is the front speed. Here we compare for first time theoretical predictions for the speed of RD fronts with 


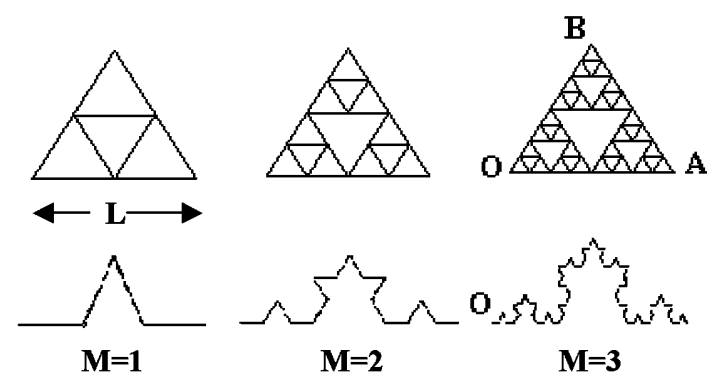

Fig. 1 - The iteration process for the SG (top) and the $\mathrm{KC}$ (bottom) as a function of the level $M$.

simulations on fractal structures and discuss the properties of these simulated fronts. For this purpose, we have used two deterministic finitely ramified [13] fractals: the SG and the KC. The reason for this choice is that deterministic fractals, as we will show, allow us to perform an extremely simple analysis based on their widely known properties. Finally, we include in our comparative study the CMF equation derived in [6], which is expected to hold for a large class of fractals, including random structures as percolation clusters.

The Sierpinski gasket. - The iteration process for the Sierpinski-gasket construction can be seen in fig. 1 (top). We will consider walks starting from the origin $O$ so that at any time step $\tau$ the walkers have the same probability of jumping to every one of their first neighbours (in the SG, all points have 4 first-neighbours, except for the origin $O$ ). Then, going up to the regime $t \gg \tau$, we can consider the process as continuous.

To analyze walks within this complex structure, we will make the assumption that diffusion through the chemical-distance space behaves as classical diffusion [8]. The chemical distance $l$ is defined as the minimum distance between two points within the fractal (it is the minimum number of points that a particle must visit to go from one of the points to the other one). This equivalence ( $l$-distance is equal to the number of jumps required) is what led us to consider that diffusion through $l$-space behaves in some sense as classical diffusion (we shall see below the limitations of this assumption). According to this, we can write the evolution of the probability density $n(l, t)$ to find a particle at time $t$ at a distance $l$ from the origin, as

$$
\frac{\partial n}{\partial t}=D \frac{\partial^{2} n}{\partial l^{2}}+a n(1-n)
$$

which is the classical diffusion equation plus a growth (logistic) function. $D$ is the diffusion coefficient and $a$ is the growth parameter, which here is taken as a constant, as usual. Travelling solutions of eq. (1) have been widely studied before $[14,15]$ and it is known that they yield propagative fronts with velocity

$$
v_{l}=\frac{\mathrm{d} l}{\mathrm{~d} t}=2 \sqrt{a D}
$$

Looking at eq. (2), we conclude that we just need to estimate the parameter of diffusion $D$, which in the homogeneous case has the form $[16,17] D=\frac{\left\langle\Delta l^{2}\right\rangle}{2 d \tau}$ (where $d$ is the spatial dimension), in order to find the speed through the SG in the chemical-distance space. However, this expression for $D$ is only valid if one considers the classical diffusion equation $n_{t}=D n_{l l}$ in the whole real space. There, a Gaussian solution arises and by means of the normalization $1=\int_{0}^{\infty} n(l, t) \mathrm{d} l$ the relation between $\left\langle\Delta l^{2}\right\rangle$ and $D$ is obtained [17]. Here the situation is 

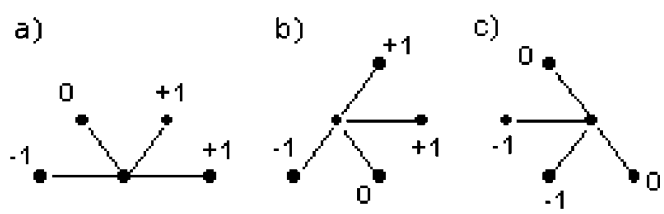

Fig. 2 - Different kinds of points in the SG with their first neighbours. The numbers in the branches correspond to the value of $\Delta l$ relative to that jump, i.e. change in $l$ from the origin.

different and we must integrate only over the fractal structure. As shown before $[3,6]$, the normalization rule for fractals reads $1=\int_{0}^{\infty} d_{l} n(l, t) l^{d_{l}-1} \mathrm{~d} l$. It arises from the fact that the number of points in the structure grows as $l^{d_{l}}$ (this relation defines the exponent $d_{l}$, which is equivalent in the $l$-space to the usual fractal dimension $d_{f}$ in the Euclidean space), so the integration is over $\mathrm{d}\left(l^{d_{l}}\right)=d_{l} l^{d_{l}-1} \mathrm{~d} l[3]$. This normalization rule leads us to

$$
D=\frac{\left\langle\Delta l^{2}\right\rangle}{2 d_{l} \tau} .
$$

So, eq. (3) is the form of the diffusion coefficient $D$ for fractals assuming that eq. (1) is valid.

Now, the way to find $\left\langle\Delta l^{2}\right\rangle$ for the SG case is by analyzing the different kind of points within that structure (fig. 2). From arguments of symmetry, it is easy to see that the SG is made up by one third of points of each class, a), b) and c). In fig. 2 we also represent the distances $\Delta l$ due to the jumps to the 4 first neighbours. According to the arguments above about $l$, in the direction $A B$ (in fig. 1) all the points have the same value of $l$, so a jump in this direction means $\Delta l=0$. All this leads us to estimate $D$ from (3) as

$$
D=\frac{1}{2 d_{l} \tau}\left(\frac{1}{3} \frac{3 l_{0}^{2}}{4}+\frac{1}{3} \frac{3 l_{0}^{2}}{4}+\frac{1}{3} \frac{2 l_{0}^{2}}{4}\right) \simeq 0.21 \frac{l_{0}^{2}}{\tau},
$$

where $l_{0}=L / 2^{M}$ is the distance between first neighbours (for simulations, we will always take $l_{0}=1$ and $\tau=1$ for simplicity). Moreover, we have used the relation $d_{l}=d_{f}=1.58$ for the $\mathrm{SG}[2]$. In consequence, the predicted speed will be

$$
v_{l} \simeq 0.92 \sqrt{\frac{l_{0}^{2} a}{\tau}} .
$$

For now, we have found an expression for the speed in the $l$-space, but we are usually more interested in the Euclidean space, defined by the distance $r$. Passing from one space to the other is really very simple by means of another well-known power law relation [2]

$$
l=k r^{d_{\min }}
$$

where $k$ is a constant and $d_{\min }$ is called the minimum-distance dimension. When we introduce the new variable $r$ into eqs. (1) and (2), they turn into

$$
\begin{aligned}
\frac{\partial n}{\partial t} & =D \frac{r^{1-d_{\min }}}{k d_{\min }} \frac{\partial}{\partial r}\left(\frac{r^{1-d_{\min }}}{k d_{\min }} \frac{\partial n}{\partial r}\right)+a n(1-n), \\
v_{r} & =\frac{\mathrm{d} r}{\mathrm{~d} t}=\frac{1}{d_{\min }}\left(\frac{4 a D}{k^{2}}\right)^{\frac{1}{2 d_{\min }}} t^{\frac{1}{d_{\min }}}-1
\end{aligned}
$$




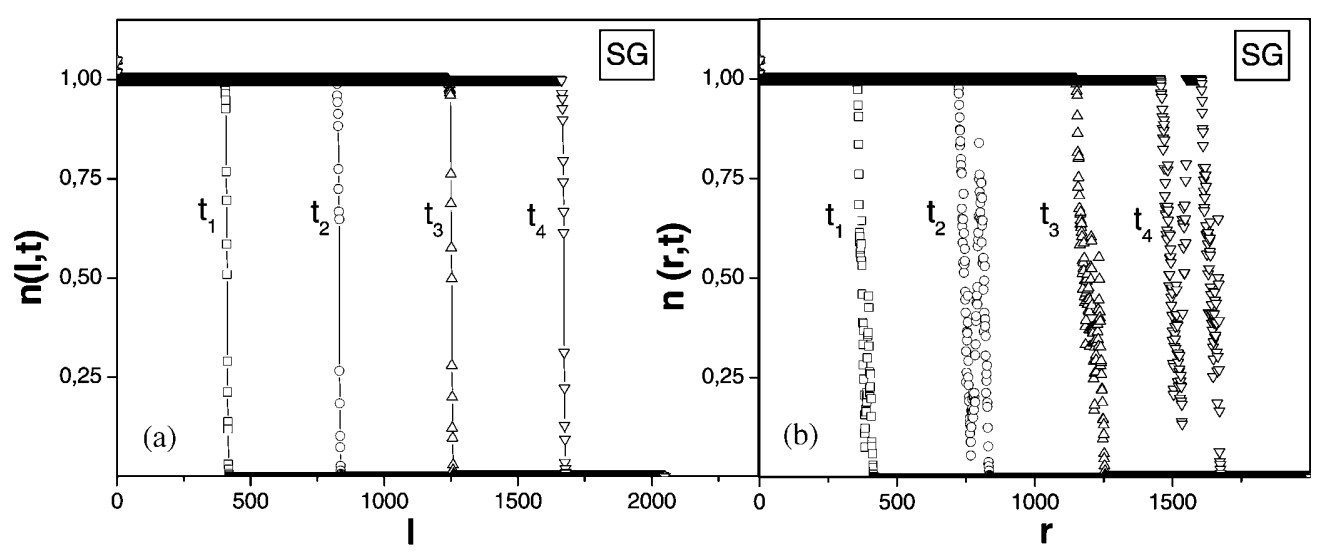

Fig. 3 - Typical fronts found on the SG $(M=11)$ for different times $\left(t_{1}<t_{2}<t_{3}<t_{4}\right)$ as a function of (a) the chemical distance and (b) the Euclidean distance from the origin.

The values of $k$ and $d_{\min }$ for the SG can be found from simulations of eq. (6), which yield $k=1.01 \pm 0.01$ and $d_{\min }=1.01 \pm 0.01$. This leads us to the conclusion that $l \approx r$ for the SG, so $v_{r} \approx v_{l}$ should be expected.

The plot in fig. 3 shows the evolution of the travelling front arising from random-walk simulations as those described above on the SG and then adding the reaction term by applying the growth function $a n(1-n)$ to every point of the lattice at any time step. In the $l$-space, we obtain step-like fronts, as happens in RD processes in homogeneous media, but for the Euclidean space the fronts present a certain width which grows with time. This is due to the relation (6): the more walkers advance through the fractal and find greater obstacles, the more fluctuations on the averaged behavior (6) grow; this is an aspect that must be taken into account to study fronts on fractals, as noted before by some authors [18].

The Koch curve. - The KC is shown in fig. 1 (bottom). It is a topologically 1D or "linear" fractal (which means $d_{l}=1[2]$ ), so it should be expected that transport processes on it were easier to describe. In fact, in the $l$-space, diffusion through the $\mathrm{KC}$ is analogous to diffusion through a one-dimensional chain, so in this case our assumption for the $l$-space follows exactly. The form of the diffusion coefficient, similar to that done in eq. (4) (but now $l_{0}=L / 3^{M}$ ), is

$$
D=\frac{1}{2 d_{l} \tau}\left(\frac{l_{0}^{2}}{2}+\frac{l_{0}^{2}}{2}\right)=\frac{l_{0}^{2}}{2 \tau}
$$

As diffusion in $l$-space is trivial in this case, we can directly go to study the $r$-space. There, eqs. (7) and (8) should hold, as the arguments for the SG are still valid. The parameters $k$ and $d_{\text {min }}$ are taken again from simulations of $r$ vs. $l$. We obtain in this case $k=1.08 \pm 0.02$ and $d_{\min }=1.27 \pm 0.01$. This is in agreement with the fact that for $1 \mathrm{D}$ topological structures $d_{\text {min }}$ must equal the fractal dimension $d_{f}$ (for the $\mathrm{KC}, d_{f}=\ln 4 / \ln 3=1.26$ ) [2]. These results will serve us to predict the speed $v_{r}$ and to compare it with the speed of the simulated fronts. This has been done in fig. 4 (choosing the point $O$ in fig. 1 as the origin), where one can see that the agreement is excellent.

It is important to note that, although the $\mathrm{KC}$ is expected to yield a simpler dynamics than the SG (due to the fact that it is topologically linear), we find in this case that fronts are decelerated (see fig. 4). The reason is that the only one parameter determining the acceleration 


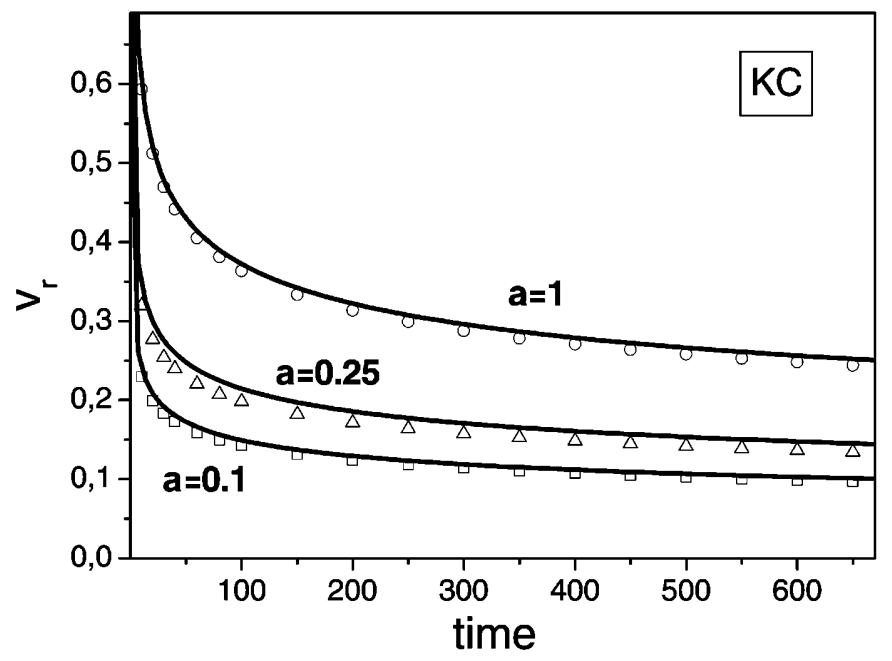

Fig. 4 - Averaged speed found from RD simulations (points) on the $\mathrm{KC}$ when $M=9$ and from the theory (lines) as a function of time and for different values of $a$. All parameters plotted are adimensonal.

of fractal fronts is $d_{\text {min }}$, as we have emphasized in previous works [8]. From eq. (8) it can be concluded that fronts in fractals are decelerated except when $d_{\min }=1$. This is what happens for the SG case shown before, where fronts advance with constant speed.

We want to stress that most numerical studies on fractal diffusion have just focused on the SG case. Then, the effect of $d_{\text {min }}$ has not been shown before. Our work shows that it is important to consider this parameter, as its effect is essential for the dynamics of fronts.

Comparison with the CMF equation. - The CMF equation has the form

$$
\frac{\partial n}{\partial t}=\frac{4 D_{0}}{d_{w}^{2} r^{d_{f}-1}} \frac{\partial}{\partial r}\left[\left(\frac{r}{t^{1 / d_{w}}}\right)^{d_{w}-u} r^{d_{f}-d_{w}+1} \frac{\partial n}{\partial r}\right]+a n(1-n)
$$

where $u=d_{w} d_{\min } /\left(d_{w}-d_{\min }\right), d_{w}$ is the fractal dimension of the random walk [2] and $D_{0}$ is the fractal-diffusion coefficient, whose value can be estimated from

$$
\left\langle r^{2}\right\rangle=\frac{\Gamma\left[\left(d_{f}+2\right) / u\right]}{\Gamma\left[d_{f} / u\right]}\left(\frac{4 u D_{0}}{d_{w}}\right)^{\frac{2}{u}} t^{\frac{2}{d_{w}}},
$$

which arises directly from the normalization of the solution in (10) [6].

We recently proved that (10) is the only PDE proposed to date which can reproduce the best-known asymptotic results on fractal diffusion $[6,8]$. According to this, the front speed predicted by it,

$$
v_{r}=\frac{1}{d_{\min }}\left(\frac{4 a D_{0} d_{\min }}{d_{w}-d_{\min }}\right)^{\frac{d_{w}-d_{\min }}{d_{w} d_{\min }}} t^{\frac{1}{d_{\min }}-1},
$$

should agree with that from simulations on the SG and the KC. Figure 5, which summarizes the main results obtained from our work, shows the comparison between the simulations (points), the CMF equation (solid lines) and eq. (7) presented here for first time (dotted lines), as a function of the growth rate $a$, which is the only one parameter absolutely independent of the 


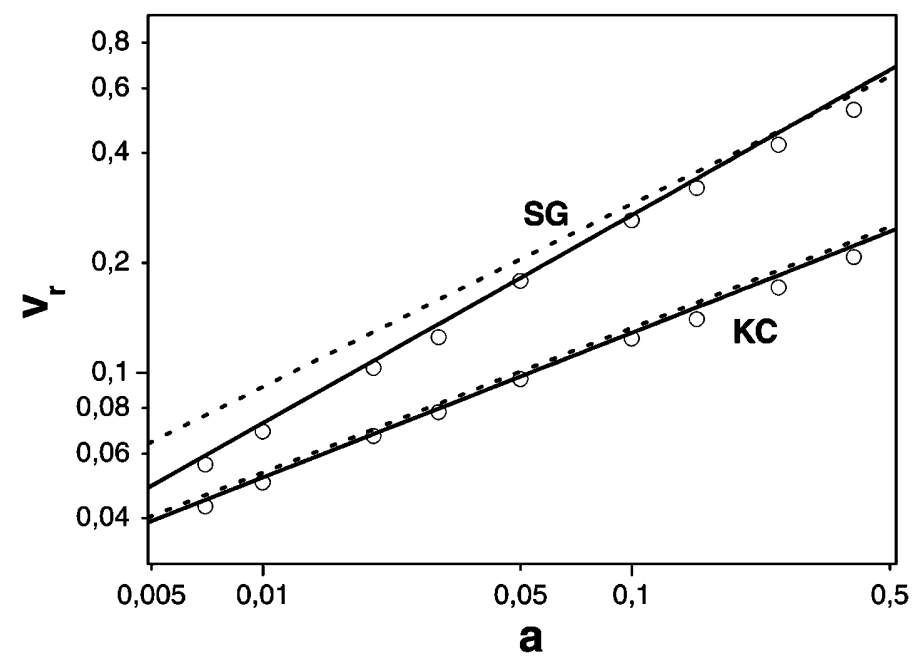

Fig. 5 - Comparison between the theoretical speed for the model presented here (dotted lines), the CMF (solid lines) and simulations (points) as a function of $a$ (the log-log scale allows us to show different orders of magnitude of $a$ ). For the KC (where fronts are accelerated) we chose arbitrarily $t=200$. All parameters plotted are adimensional.

fractal structure considered [19]. In the case of $\mathrm{KC}$, as the front is decelerated, we have used for the plot a fixed arbitrary time $t=200$.

The agreement found is good in general, so this confirms that our two approximations are suitable for the description of RD fronts through these fractals. However, we observe some deviations in the SG case for the $l$-space model, so we should discuss about the validity of the model presented, based on the assumption that diffusion in the $l$-space shows classical behavior. We have already explained above that this assumption is correct for 1D topological structures. There, $d_{l}=1$ and so fractal effects do not appear for $l$ (the diffusion in the $l$-space is exactly analogous to non-fractal diffusion in 1D). Hence, the small deviations found for the $\mathrm{KC}$ case (fig. 5) can only be due to the statistical fluctuations involved in the relation (6).

For the SG, the agreement found is not so good, so at the sight of this we can conclude that the assumption for $l$ is less accurate for non-linear topological structures; for those cases, a more general approach is advisable. However, fig. 5 shows that this method can fit approximately the propagation rate of fronts also for the SG, so eq. (8) can be still useful in this case.

In contrast with that, the CMF equation should hold in principle for most fractals $[6,8]$, including random structures. Nevertheless, it is a more complex approach than (7), since it involves more parameters. Here we have tried to present a very simple model based on analogies with classical transport processes, and compare it to the more complicate CMF equation in order to show its validity.

Anyway, we do not mean that the equations studied here are able to explain all the intrincate features of such a complex process as transport in fractals. They are just an averaged (asymptotic) approximation, since scaling relations in fractals, which are in the background of our work, are just statistical laws. Actually, multifractality and some complex characteristics of fractals dynamics observed before $[2,20]$ are not considered here. So, we want to stress that our approach, in spite of its interest for fractal theory, is rather a predictive tool useful for those experimentalists working on transport through self-similar media. 
DC acknowledges the DURSI of the Generalitat de Catalunya. Partially funded by the Generalitat de Catalunya under grant SGR-2001-00186, and by the MICYT under grants REN-2003-00185 CLI (DC and JF) and BFM-2003-06033 (VM).

\section{REFERENCES}

[1] Havlin S., Ben-Avraham D. and Sompolinsky H., Phys. Rev. A, 27 (1983) 1730; Guyer R. A., Phys. Rev. A, 32 (1984) 2324; Havlin S., Movshovitz D., Trus B. and West G. H., J. Phys. A, 18 (1985) L719.

[2] Ben-Avraham D. and Havlin S., Diffusion and Reactions in Fractals and Disordered Systems (Cambridge University Press, Cambridge) 2000.

[3] O'Shaugnessy B. and Procaccia I., Phys. Rev. Lett., 54 (1985) 455; Phys. Rev. A, 32 (1985) 3073.

[4] Giona M. and Roman H. E., Physica A, 185 (1992) 87.

[5] Metzler R. et al., Physica A, 211 (1994) 13.

[6] Campos D., Méndez V. and Fort J., Phys. Rev. E, 69 (2004) 031115.

[7] Fedotov S. and Méndez V., Phys. Rev. E, 66 (2002) 030102(R).

[8] Méndez V., Campos D. and Fort J., Phys. Rev. E, 69 (2004) 016613.

[9] Giménez D. et al., Eng. Geol., 48 (1997) 161.

[10] Liu Y., Hou Z., Hui P. M. and Sritrakool W., Phys. Rev. B, 60 (1999) 13444; Bhattacharyya B. and Chakrabarti A., Phys. Rev. B, 58 (1998) 2376.

[11] Caldarelli G. et al., Europhys. Lett., 56 (2001) 510.

[12] Johnson A. R., Milne B. T. and Wiens J. A., Ecology, 73 (1992) 1968.

[13] Diffusion on infinitely ramified fractals is a field where very little research has been done. Fractals in nature are usually finitely ramified, so they are more suitable for the aims of our work.

[14] Kolmogorov A. N., Petrovski I. G. and Piskunov N. S., Bull. Univ. Moscow A, 1 (1937) 1.

[15] Murray J. D., Mathematical Biology (Springer, Berlin) 1993.

[16] Fort J. and Méndez V., Phys. Rev. Lett., 82 (1999) 867.

[17] Cranck J., The Mathematics of Diffusion (Oxford University Press, London) 1956.

[18] Barabási A.-L. and Stanley H. E., Fractal Concepts in Surface Growth (Cambridge University Press, Cambridge) 1995.

[19] Sierpinski gasket: $d_{f}=1.58, d_{w}=2.32$; Koch curve: $d_{f}=1.27, d_{w}=2.53$. Values from [2].

[20] Bunde A. and Dräger J., Phys. Rev. E, 52 (1995) 53. 